TITLE:

\title{
The effects of high-frequency ultrasound on turbulent liquid mixing with a rapid chemical reaction
}

$\operatorname{AUTHOR}(S):$

Ito, Y; Nagata, K; Komori, S

\section{CITATION:}

Ito, Y ... [et al]. The effects of high-frequency ultrasound on turbulent liquid mixing with a rapid chemical reaction. PHYSICS OF FLUIDS 2002, 14(12): 4362-4371

ISSUE DATE:

2002-12

URL:

http://hdl.handle.net/2433/39781

\section{RIGHT:}

Copyright 2002 American Institute of Physics. This article may be downloaded for personal use only. Any other use requires prior permission of the author and the American Institute of Physics. 


\title{
The effects of high-frequency ultrasound on turbulent liquid mixing with a rapid chemical reaction
}

\author{
Yasumasa Ito, Kouji Nagata, and Satoru Komoria) \\ Department of Mechanical Engineering, Kyoto University, Kyoto 606-8501, Japan
}

(Received 10 January 2002; accepted 9 September 2002; published 6 November 2002)

\begin{abstract}
The effects of high-frequency ultrasound and mean fluid shear on turbulent mixing with a rapid chemical reaction were experimentally investigated in three types of liquid mixing-layer flow downstream of a turbulence-generating grid; pure grid-generated turbulence, grid-generated turbulence with high-frequency ultrasonic irradiation, and grid-generated turbulence with mean fluid shear. Instantaneous velocity and concentration were simultaneously measured using the combination of a laser-Doppler velocimeter and a laser-induced fluorescence method. The results show that turbulent mixing and chemical reaction are promoted by ultrasonic irradiation and mean fluid shear. The amount of chemical product in grid-generated turbulence with high-frequency ultrasonic irradiation is much larger than that in grid-generated turbulence with mean fluid shear, despite turbulent mass transport being enhanced at an equivalent level in both flows. This is attributed to the difference in turbulent mass transport at small scales. Ultrasonic irradiation more enhances the mass transport at smaller scales than by mean fluid shear, whereas mean shear can promote the mass transport only at larger scales. As a result, high-frequency ultrasound can be regarded as a better tool for promoting turbulent mixing and chemical reaction than mean fluid shear. (C) 2002 American Institute of Physics. [DOI: 10.1063/1.1518508]
\end{abstract}

\section{INTRODUCTION}

Turbulent reacting flows can be seen in many industrial plants such as chemical reactors and in the atmospheric boundary layer with turbulent diffusion of reactive contaminants like $\mathrm{NO}_{X}$ and $\mathrm{SO}_{X}$ exhausted from automobiles or power plants. It is, therefore, of great importance in fluid and environmental engineerings to study the turbulent reactivediffusive mechanism and to propose a method to control (promote or retard) the progress of the chemical reaction.

When two nonpremixed reactants, $A$ and $B$, are introduced into a turbulent flow, the chemical reactants are convected and mixed mainly by turbulent motions, and the chemical reaction proceeds through molecular diffusion at the complicatedly deformed interface between two reactive fluids. Therefore the turbulence structure plays an important role in the progress of chemical reaction until two reactants are perfectly mixed up to the minimum scale of turbulence: the Batchelor scale. ${ }^{1}$ This means that it is significant in promoting chemical reaction in a nonpremixed turbulent reacting flow to intensively deform the interface between two reactive fluids at small scales.

One of the promising methods for modifying turbulence structure and promoting chemical reaction is the use of mean fluid shear. ${ }^{1}$ Mean fluid shear is usually generated by velocity difference between two reactive fluids. But experiments indicate that the mean fluid shear enhances turbulent mixing mainly at large scales but not at small scales that play an important role in the progress of chemical reaction. Recently

\footnotetext{
a) Author to whom correspondence should be addressed. Telephone: +81-75753-5244; electronic mail: komori@mech.kyoto-u.ac.jp
}

Nagata and Komori ${ }^{2}$ experimentally investigated the effects of unstable stratification and mean fluid shear on turbulent mixing and chemical reaction. They concluded that the turbulent mixing is enhanced at both large and small scales by buoyancy under unstable, density-stratified conditions. The buoyancy-driven mixing promoted the chemical reaction more vigorously than the sheared mixing with an equivalent turbulence level. The results suggest that chemical reaction in a nonpremixed turbulent reacting flow is effectively promoted by applying a technique that can enhance turbulent mixing at both large and small scales. In the present study, we propose the use of high-frequency ultrasound as a new technique for promoting turbulent mixing and chemical reaction.

It is well known that when ultrasound is irradiated to liquids, two phenomena, acoustic cavitation and acoustic streaming, appear in the liquids. The acoustic cavitation associated with bubbles ${ }^{3-6}$ is generated by intense pressure fluctuations in the ultrasonic field, and low-frequency ultrasound with frequency less than $100 \mathrm{kHz}$ is much more effective for generating cavitation than high-frequency ultrasound. The acoustic streaming is a steady flow generated by energy gradient of the ultrasonic wave. There are two types of acoustic streaming, depending on the frequency of the ultrasound. One is Rayleigh streaming. ${ }^{3,4,7}$ This streaming can be observed in a stable standing wave field which is generated by a low-frequency ultrasonic irradiation in static liquids. It is a vortex-like flow and the scales of the flow are smaller than the wavelength of the ultrasound. The other is Eckart streaming. ${ }^{8-10}$ The scales are larger than the wavelength of the ultrasound in this case, which can usually be seen when high-frequency ultrasound is irradiated to liquids. 


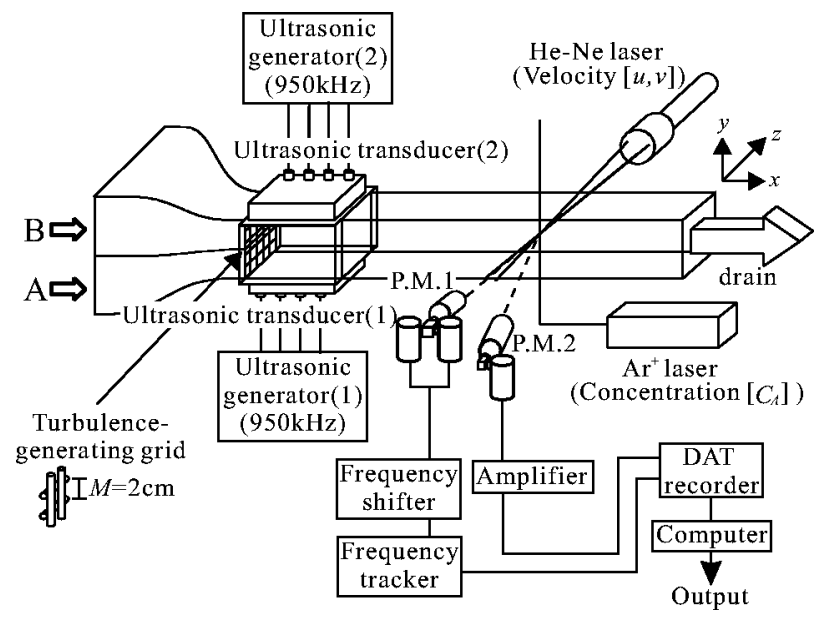

FIG. 1. Schematic of the experimental apparatus and measuring system.

Many previous studies have reported that mass transfer in liquids is promoted by ultrasonic irradiation. However, these studies on the effects of ultrasound on mass transfer have mainly dealt with static fluids, ${ }^{3-6,8-10}$ and the effects of ultrasound in laminar or turbulent flow have scarcely been investigated. In particular no studies have referred to the turbulent mixing and reactive-diffusive mechanism under ultrasonic irradiation. Indeed, the effects of ultrasound on chemical reactions have been studied in the field of sonochemistry, but these studies have aimed to investigate the effects of the particular phenomena in relation to acoustic cavitation under low-frequency ultrasonic irradiation, not to clarify the reactive-diffusive mechanism in the fluids. ${ }^{4-7}$ Although some studies have reported that a large-scale flow is originated when strong low-frequency ultrasound is irradiated to static liquids, they have considered the large-scale flow in relation to the motions of the bubbles in acoustic streaming. ${ }^{5-7}$ To investigate the effects of ultrasound on turbulent mixing and reactive-diffusive mechanism, we have to distinguish the two effects of acoustic streaming and acoustic cavitation. For high-frequency ultrasound, which generates little acoustic cavitation, some studies investigated the effects on mass transfer in initially static liquids, ${ }^{8-10}$ but they have not dealt with the effects on chemical reaction.

The purpose of this study is to clarify the effects of high-frequency ultrasonic irradiation on turbulent mixing and chemical reaction in a reacting liquid flow. In this study, we experimentally investigate the turbulence structure under high-frequency ultrasonic irradiation, which generates strong acoustic streaming of the Eckart type and little acoustic cavitation, and we discuss the effectiveness of high-frequency ultrasound as a tool for promoting turbulent mixing and chemical reaction in comparison with the promoting effects of mean fluid shear.

\section{EXPERIMENTS}

Figure 1 shows the schematic of the experimental apparatus and measuring system. The test apparatus used was a rectangular water tunnel made of polymethylemethacrylate (PMMA), $1.5 \mathrm{~m}$ in length and $0.1 \times 0.1 \mathrm{~m}$ in cross-section. A
TABLE I. Experimental conditions.

\begin{tabular}{|c|c|c|c|c|}
\hline Run & $\begin{array}{c}\overline{U_{a}} \\
{[\mathrm{~m} / \mathrm{s}]}\end{array}$ & $\begin{array}{c}P_{U} \\
{[\mathrm{~W}]}\end{array}$ & $\begin{array}{c}\Delta \bar{U} \\
{[\mathrm{~m} / \mathrm{s}]}\end{array}$ & Flow type \\
\hline I-NR & $12.5 \times 10^{-2}$ & 0 & 0 & Nonreacting \\
\hline I-R & $12.5 \times 10^{-2}$ & 0 & 0 & Reacting \\
\hline II-NR & $12.5 \times 10^{-2}$ & 80 & 0 & Nonreacting \\
\hline II-R & $12.5 \times 10^{-2}$ & 80 & 0 & Reacting \\
\hline III-NR & $12.5 \times 10^{-2}$ & 0 & $4.0 \times 10^{-2}$ & Nonreacting \\
\hline III-R & $12.5 \times 10^{-2}$ & 0 & $4.0 \times 10^{-2}$ & Reacting \\
\hline
\end{tabular}

turbulence-generating grid was installed at the entrance to the test section, of round-rod, square-mesh, single-biplane construction. The mesh size, $M$, and diameter of the rod, $d$, were $2.0 \times 10^{-2} \mathrm{~m}$ and $3.0 \times 10^{-3} \mathrm{~m}$, respectively.

The experimental conditions are listed in Table I. The experiments were carried out in three types of flow. The first type is typical grid-generated turbulence without any body force (hereinafter referred to as the pure GT: Run I). The flow was completely separated by a splitter plate into upper and lower streams from the reservoir tanks until the entrance to the test section. The mean velocities of the upper and lower streams, $\overline{U_{1}}$ and $\overline{U_{2}}$, were set to the same value of $0.125 \mathrm{~m} \mathrm{~s}^{-1}$, and the temperature of both the streams was set to $296 \mathrm{~K}$. The Reynolds number based on the mesh size $M$ $(=0.02 \mathrm{~m})$ and cross-sectionally averaged velocity $\overline{U_{a}}, \operatorname{Re}_{M}$, was 2500 . The effects of the splitter plate on turbulent motions were confirmed to be negligibly small by comparing the turbulence intensities between two cases with and without the splitter plate.

The second flow type is grid-generated turbulence with high-frequency ultrasonic irradiation (hereinafter referred to as the GT with ultrasound: Run II). The ultrasonic transducers (KAIJO 7857T) are installed at both the bottom $(y / M$ $=-2.5)$ and top $(y / M=2.5)$ walls of the test section in the streamwise region of $x / M=3.3-11.5$, so the ultrasound was irradiated from the both walls. The frequency of the ultrasound, $F_{U}$, was $950 \mathrm{kHz}$, and the electrical input for each ultrasonic generator (KAIJO 68101) was 80 W $(3.6$ $\times 10^{3} \mathrm{~W} \mathrm{~m}^{-2}$ ). In this condition, few bubbles were produced by acoustic cavitation, and the diameters of bubbles estimated by flow visualization were less than $0.1 \mathrm{~mm}$. When the relative velocity between a bubble and fluid was set to the maximum value; the terminal velocity of $1.0 \mathrm{~cm} / \mathrm{s}$, the Reynolds number based on the relative velocity, $\mathrm{Re}_{p}$, was about 1 . Therefore, for such small Reynolds numbers, the effect of bubbles on fluid motion was neglected. ${ }^{11}$ The thermal effect of the present low-power ultrasound was also negligibly small. The mean velocities of the upper and lower streams, $\overline{U_{1}}$ and $\overline{U_{2}}$, were set to the same value of $0.125 \mathrm{~m} \mathrm{~s}^{-1}$, as in the pure GT (Run I).

The third flow is the mixing-layer type of sheared flow with grid-generated turbulence (hereinafter referred to as the GT with mean shear: Run III). The initial velocity difference between the upper and lower streams, $\Delta \bar{U}\left(=\overline{U_{1}}-\overline{U_{2}}\right)$, was $0.04 \mathrm{~m} \mathrm{~s}^{-1}\left(\overline{U_{1}}=0.145 \mathrm{~m} \mathrm{~s}^{-1}\right.$ and $\left.\overline{U_{2}}=0.105 \mathrm{~m} \mathrm{~s}^{-1}\right)$. The value of the velocity difference was chosen to set the amount of vertically transported reactant across the horizontal plane 
TABLE II. Combinations of species $A$ and $B$.

\begin{tabular}{ccc}
\hline \hline Flow type & Species $A$ & Species $B$ \\
\hline Nonreacting flow & $\mathrm{H}_{2} \mathrm{O}$ & $\mathrm{H}_{2} \mathrm{O}$ \\
& & + \\
& & uranine \\
Reacting flow & $\mathrm{CH}_{3} \mathrm{COOH}$ & $\mathrm{NH}_{4} \mathrm{OH}$ \\
& + & + \\
& uranine & uranine \\
\hline \hline
\end{tabular}

of $y / M=0$ to the same value as that in the GT with ultrasound (Run II). Since the cross-sectionally averaged velocity, $\overline{U_{a}}$, was set to the same value of $0.125 \mathrm{~m} \mathrm{~s}^{-1}$, the Reynolds number, $\mathrm{Re}_{M}$, in the GT with mean shear (Run III) was 2500 , as in the Runs I and II.

The combinations of species $A$ in the lower layer and $B$ in the upper layer are listed in Table II. For nonreacting flow, fresh water without any chemical species was used as species $A$, and an aqueous solution with sodium fluorescein dye, uranine $\left(\mathrm{C}_{20} \mathrm{H}_{10} \mathrm{Na}_{2} \mathrm{O}_{5}\right)$, was used as species $B$. For reacting flow, acetic acid $\left(\mathrm{CH}_{3} \mathrm{COOH}\right)$ and ammonium hydroxide $\left(\mathrm{NH}_{4} \mathrm{OH}\right)$ was used as species $A$ and $B$, respectively. The neutralization between the weak acid and the weak base is expressed as an irreversible second-order rapid reaction

$$
\mathrm{CH}_{3} \mathrm{COOH}+\mathrm{NH}_{4} \mathrm{OH} \rightarrow \mathrm{CH}_{3} \mathrm{COONH}_{4}+\mathrm{H}_{2} \mathrm{O} .
$$

The reaction rate constant, $k$, was of the order $10^{8} \mathrm{~m}^{3} \mathrm{~mol}^{-1} \mathrm{~s}^{-1}$. The Damköhler number, $D a_{L}\left(=\tau_{1} / \tau_{3}\right)$, which is the ratio of the time scale of turbulent diffusion, $\tau_{1}\left(=L /\left(q^{2}\right)^{1 / 2}\right)$, to that of the chemical reaction, $\quad \tau_{3}\left(=\left(k\left(C_{A 0} C_{B 0}\right)^{-1 / 2}\right)^{-1}\right)$, was $4 \times 10^{5}$. The Damköhler number, $D a_{\lambda}\left(=\tau_{2} / \tau_{3}\right)$, defined by the ratio of the time scale of molecular diffusion, $\tau_{2}\left(=\lambda^{2} / D\right)$, to that of chemical reaction, $\tau_{3}$, was $4 \times 10^{9}$. Here $L$ is the integral length scale, $\overline{q^{2}}\left(=\frac{1}{2}\left(\overline{u^{2}}+\overline{v^{2}}+\overline{w^{2}}\right)\right)$ is the turbulence kinetic energy, $\lambda$ is the microscale and $D$ is the molecular diffusivity of mass. The values of $L$ and $\lambda$ estimated from the decay of the kinetic energy, $\overline{q^{2}}$, were 5.2 and $4.4 \mathrm{~mm}$, respectively. ${ }^{12}$ The initial concentrations of the two reacting species, $C_{A 0}$ and $C_{B 0}$, were set to the same value of $0.01 \mathrm{~mol} \mathrm{~m}^{-3}$. The uranine was homogeneously premixed into both solutions of $A$ and $B$ at the same concentration of $5.0 \times 10^{-5} \mathrm{~mol} \mathrm{~m}^{-3}$ to enable us to measure the concentration by a laser-induced fluorescence method based on the dependence of fluorescence intensity on the $\mathrm{pH}$ of the solution, ${ }^{2,13,14,16}$ as mentioned in the following paragraphs. Since the concentrations of reacting species used here were extremely low, the change of temperature due to the reaction was negligibly small. Therefore, the differences in molecular diffusivity, density, viscosity and reaction rate constant between the reacting and nonreacting cases were neglected.

Instantaneous velocities in the streamwise and vertical directions were measured in the region $14 \leqslant x / M \leqslant 20$ by a two-component laser-Doppler velocimeter (LDV). The laserDoppler velocimeter used was a DANTEC 55X Modular system with a polarization beam splitter (55X24), a $40 \mathrm{MHz}$ Bragg cell and a beam expander, and the laser was a $5 \mathrm{~mW}$
He-Ne laser (Spectra Physics model 106-1; wave length: $\lambda$ $=632.8 \mathrm{~nm}$ ). The $\mathrm{He}-\mathrm{Ne}$ laser beams were introduced from the sidewall of the test section.

Instantaneous concentrations of reacting and nonreacting species were measured using a laser-induced fluorescence (LIF) method, together with the velocities at the same measuring point. For the concentration measurement by the LIF method, the uranine diffusing in the flow was illuminated by a high-power single-line mode argon-ion $\left(\mathrm{Ar}^{+}\right)$laser (LEXEL model $95-4 ; \lambda=488 \mathrm{~nm}$ ) with $0.8 \mathrm{~W}$ power. The $\mathrm{Ar}^{+}$laser beam was introduced through the bottom wall of the test section and it was focused on a measuring point by a convex lens. The fluorescence from the measuring point was collected using the same optical system as used in Komori et al., ${ }^{2,14,16}$ and it was detected by a photomultiplier. For nonreacting flow, instantaneous concentration of the uranine which was initially premixed in the upper layer was measured as instantaneous concentration of a nonreacting species $B, C_{B}^{*}$, using a proportional calibration curve [Fig. 2(a)] between the LIF intensity and the concentration of the uranine, $C_{B}^{*}$. The reason why the uranine was premixed in the upper layer was to reduce the attenuation along the path of the laser beam introduced from the bottom wall. From the concentration of nonreacting species $B$, instantaneous concentration of nonreacting species $A, C_{A}^{*}$, was determined by $C_{A}^{*}=1-C_{B}^{*}$. For reacting flow, the uranine was initially premixed in both upper and lower layers at a constant concentration and the dependency of the LIF intensity on the $\mathrm{pH}$ of the solution was used to measure $C_{A}$. The LIF intensity from the uranine premixed at a constant concentration does not change in neutral or basic solution $(\mathrm{pH} \geqslant 7)$, but it decays depending on the concentration of the weak acid in the side of $\mathrm{pH}<7$. Therefore, a nonlinear calibration curve between the LIF intensity and the concentration of the acetic acid was obtained in a well mixed $\operatorname{tank}^{14,16}$ as shown in Fig. 2(b). By using this calibration curve, instantaneous concentration of reacting species $A$ (acetic acid), $C_{A}$, was directly measured from the LIF intensity.

Strictly, the nonlinear calibration curve [Fig. 2(b)] is applicable to the LIF method with the spatial resolution smaller than the Batchelor scale. However, it is confirmed by direct numerical simulation ${ }^{17}$ that the spatial resolution smaller than the dissipation scale is enough to estimate the turbulence statistics. The present spatial resolution was estimated to be $70 \mu \mathrm{m}$ from the power spectrum of concentration fluctuation. The resolution was larger than the Batchelor scale of about $20 \mu \mathrm{m}$ but it was much smaller than the concentration dissipation scale of about $1.1 \mathrm{~mm}$. Furthermore, the molecular mixing thickness at the measurement section of 14 $\leqslant x / M \leqslant 20$ was roughly estimated to be $100 \mu \mathrm{m}$ and the thickness was larger than the present spatial resolution. This suggests that the concentration does not steeply change within the present measuring volume and the concentration field in the measuring volume is close to well-mixed condition because of the smearing effect of molecular diffusion. Both the smearing effect and small spatial resolution enabled us to use the nonlinear calibration curve [Fig. 2(b)] for measuring $C_{A}$ in the region of $14 \leqslant x / M \leqslant 20$. The details of the 

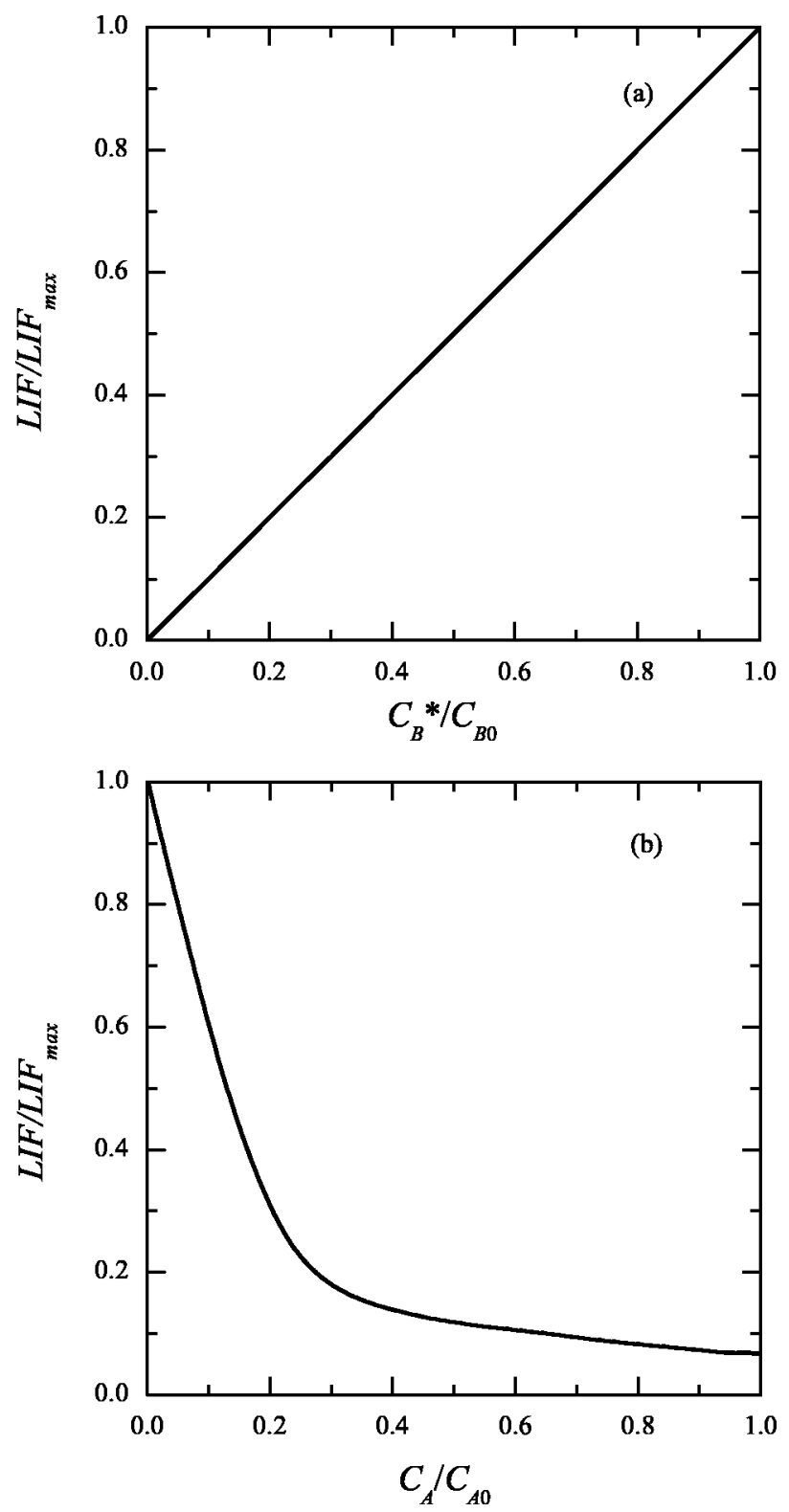

FIG. 2. Calibration curve between the normalized LIF intensity and the instantaneous concentration; (a) linear relation between LIF intensity and concentration of nonreacting species $B$; (b) nonlinear relation between LIF intensity and concentration of reacting species $A$.

measuring system have been described in previous papers. $2,14,16$

The sampling frequency and the sample size for the velocity and concentration signals were $4.0 \mathrm{kHz}$ and 240000 points, respectively. The digitized data were recorded by a data recorder (SONY PC208AX), and statistical processing of the data was performed by a computer (EPSON Endeavor Pro-400S).

For flow visualization by $\mathrm{LIF}^{15}$ the vertical $\mathrm{Ar}^{+}$-laser sheet was also introduced through the bottom wall of the test section. The flow visualization images were captured by a digital CCD camera (SONY DCR-VX2000). The images covered the area of $14 \mathrm{~cm} \times 10 \mathrm{~cm}$ corresponding to the region of $x / M=15.5-19.0$, and each image consists of $340 \mathrm{~K}$ pixels. The shutter speed of the camera was $1 / 15 \mathrm{~s}$.

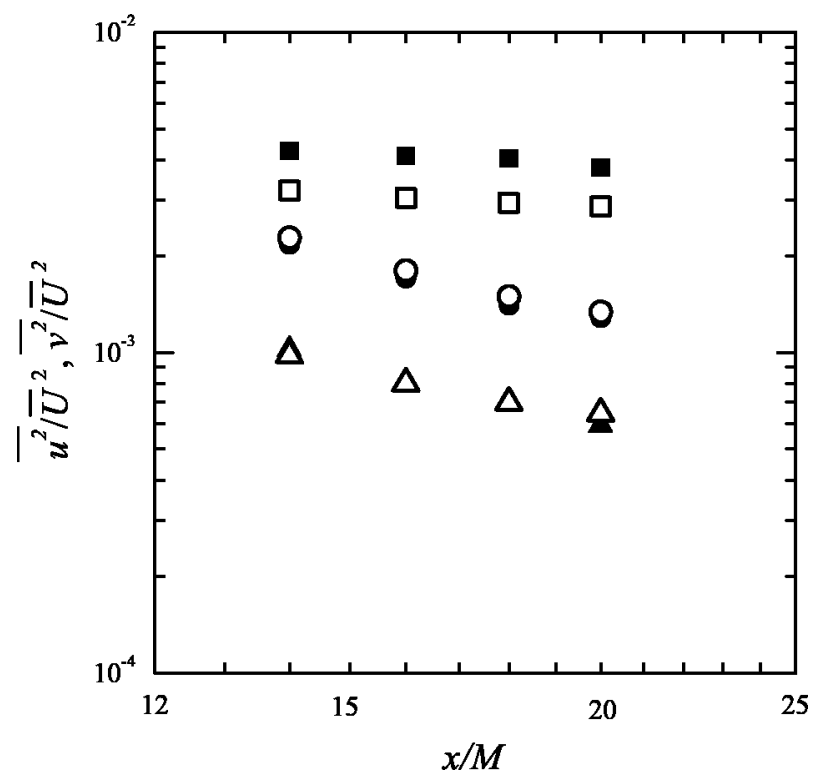

FIG. 3. Streamwise distributions of the mean-squared values of streamwise and vertical velocity fluctuations at $y / M=0.0: \mathbf{\Delta}, \overline{u^{2}} / \bar{U}^{2}$ in the pure GT (Run I); $\triangle, \overline{v^{2}} / \bar{U}^{2}$ in the pure GT (Run I); $\bullet, \overline{u^{2}} / \bar{U}^{2}$ in the GT with ultrasound (Run II); $\bigcirc, \overline{v^{2}} / \bar{U}^{2}$ in the GT with ultrasound (Run II); $\mathbf{\square}, \bar{u}^{2} / \bar{U}^{2}$ in the GT with mean shear (Run III); $\square, \overline{v^{2}} / \bar{U}^{2}$ in the GT with mean shear (Run III).

\section{RESULTS AND DISCUSSION}

Figures 3 and 4 show the streamwise and vertical distributions of the mean squared values of the streamwise velocity fluctuation and vertical velocity fluctuation, $\overline{u^{2}}$ and $\overline{v^{2}}$. Here the velocity fluctuations are normalized by the squared value of the mean velocity, $\bar{U}^{2}$. In the pure GT (Run I), the streamwise and vertical velocity fluctuations have almost the same values and decay following by a power law with an exponent of 1.47. The value of the exponent compares well with the values from the previous measurements. ${ }^{2}$ The correlation coefficient between $u$ and $v, R_{u v}=\overline{u v} / \sqrt{u^{2}} / \sqrt{v^{2}}$, was almost equal to zero in the whole region. The results show that isotropic homogeneous turbulence is developed in the flow downstream of the turbulence-generating grid, and the effects of the splitter plate installed before the grid on turbulent motions were also confirmed to be negligibly small by comparing the turbulence intensities between two cases with and without the splitter plate. On the other hand, the turbulence intensities in the streamwise and vertical directions are enhanced both in the GT with ultrasound (Run II) and in the GT with mean shear (Run III). Around the centerline of the test section, the turbulence intensities in both streamwise and vertical directions are larger in the GT with mean shear (Run III) than those in the GT with ultrasound (Run II). At $x / M=18$, in the GT with ultrasound (Run II), both the mean-squared values of the streamwise and vertical velocity fluctuations, $\overline{u^{2}}$ and $\overline{v^{2}}$, become about $1.5-2.3$ times larger than those in the pure GT (Run I). On the other hand, in the GT with mean shear (Run III), although $\overline{u^{2}}$ and $\overline{v^{2}}$ on the centerline $(y / M=0)$ where the vertical velocity gradient is large are about 5.3 times and 4.4 times larger than those in 

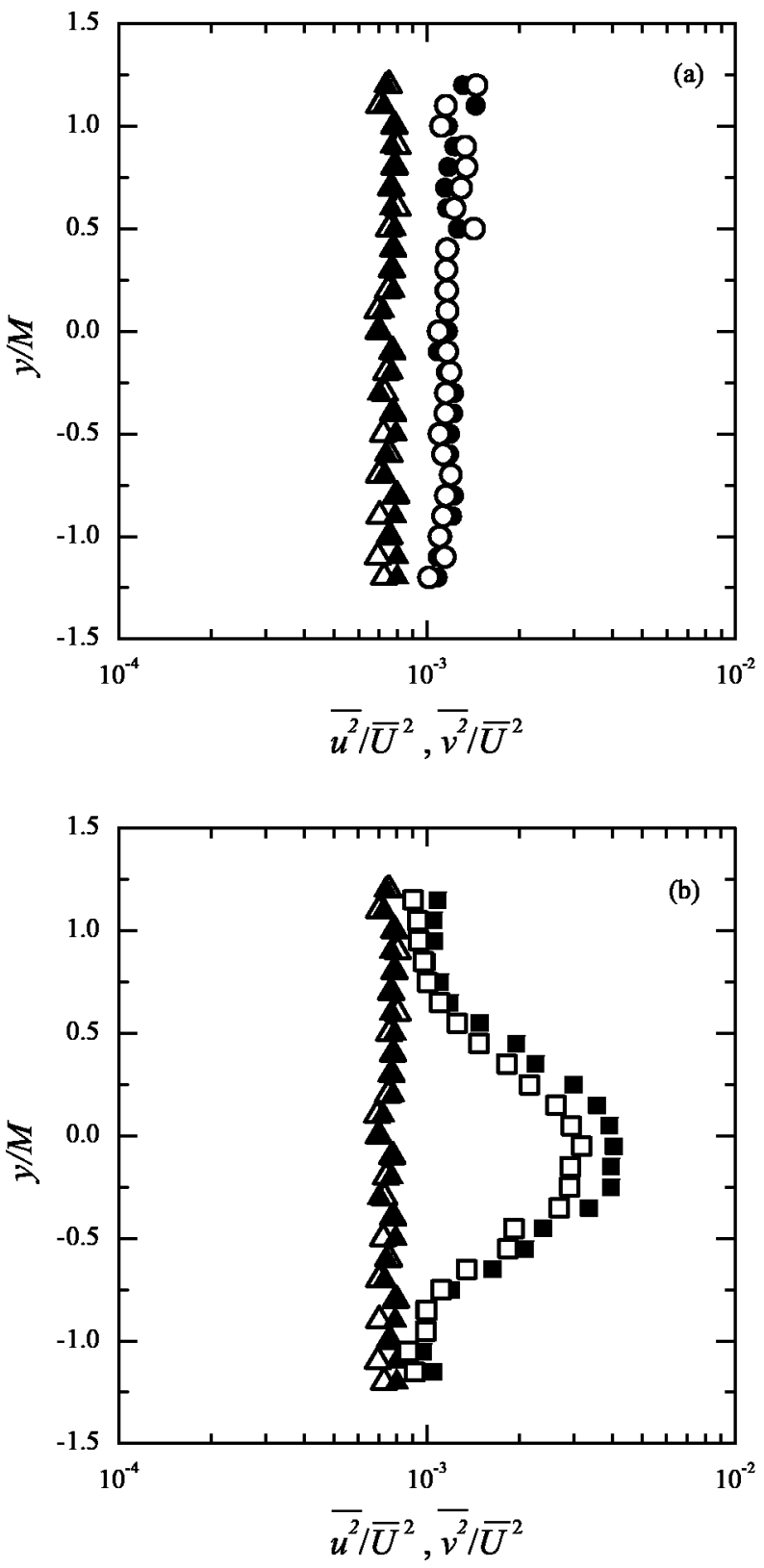

FIG. 4. Vertical distributions of the mean-squared values of streamwise and vertical velocity fluctuations at $x / M=18$ in (a) in the GT with ultrasound (Run II) and the pure GT (Run I) and (b) in the GT with mean shear (Run III) and the pure GT (Run I). Symbols as in Fig. 3.

the pure GT (Run I), respectively, the differences decrease around $y / M= \pm 1.0$, where the vertical velocity gradient is small.

A main reason of the enhancement for the turbulence intensities in the GT with ultrasound (Run II) is Eckart-type acoustic streaming. The governing equations of Eckart-type acoustic streaming are the equation of continuity and the Navier-Stokes (NS) equation in an incompressible fluid. When ultrasound is introduced vertically as in the present experiments, the NS equation in the vertical $(y)$ direction is written as

$$
\frac{\partial V}{\partial t}+(\boldsymbol{V} \cdot \nabla) V=-\frac{1}{\rho} \frac{\partial P}{\partial y}+\nu \nabla^{2} V+F_{y} .
$$

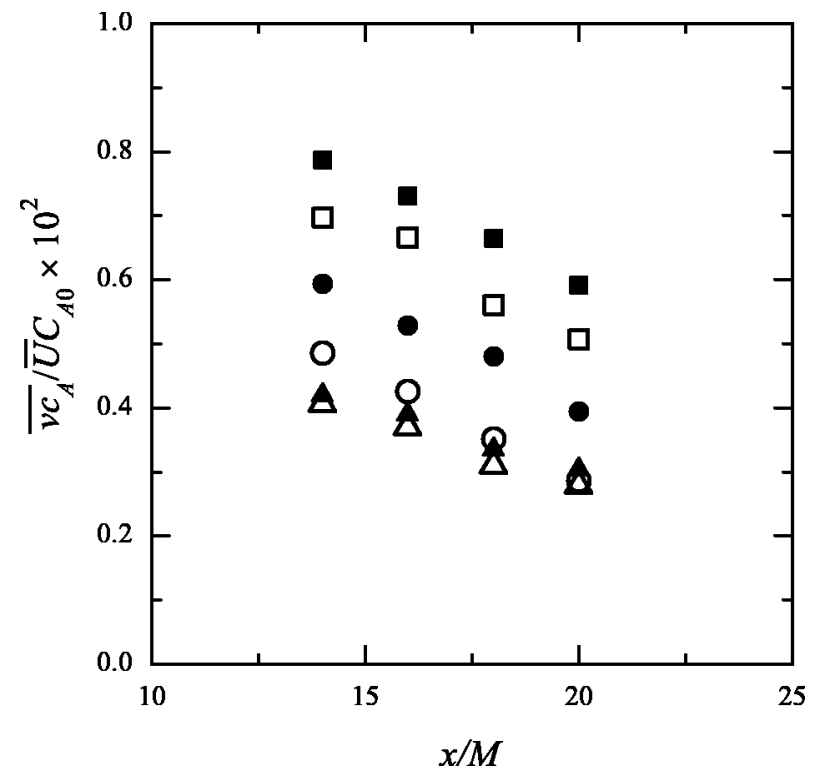

FIG. 5. Streamwise distributions of the normalized vertical mass flux of species $A$ at $y / M=0.0: \boldsymbol{\Delta}$, in the nonreacting pure GT (Run I-NR); $\triangle$, in the reacting pure GT (Run I-R); $\bullet$, in the nonreacting GT with ultrasound (Run II-NR); $\bigcirc$, in the reacting GT with ultrasound (Run II-R); $\mathbf{\square}$, in the nonreacting GT with mean shear (Run III-NR); $\square$, in the reacting GT with mean shear (Run III-R).

Here $\rho$ is the density of the static fluid, $P$ is the instantaneous pressure, $V$ is the instantaneous vertical velocity, and $F_{y}$ is the driving force of the acoustic streaming. If the ultrasonic wave is a plane wave, $F_{y}$ can be expressed as

$$
F_{y}=-\frac{1}{\rho} \frac{d E}{d y} .
$$

Here $E$ is the time-averaged energy density of the ultrasound. Equation (2) means that the energy gradient of an ultrasonic wave is the driving force of acoustic streaming. As the ultrasonic wave propagates, the acoustic intensity decreases $\left(d E / d y \leqslant 0\right.$, i.e., $\left.F_{y} \geqslant 0\right)$ owing to both the viscosity of liquid and the nonlinearity of ultrasound. This mechanism causes acoustic streaming.

Figure 5 shows the streamwise distributions of the vertical mass flux of species $A, \overline{v c_{A}}$, in the nonreacting case together with those in the reacting case. Here the fluxes are normalized by the mean velocity $\bar{U}$ and the initial concentration of species $A, C_{A 0}$. The vertical mass transport in the GT with mean shear (Run III) is stronger than that in the GT with ultrasound (Run II) in the whole region. However, the difference in $\overline{v c_{A}}$ between the nonreacting and reacting cases is larger in the GT with ultrasound (Run II) than that in the GT with mean shear (Run III). This means that the chemical reaction in the GT with ultrasound (Run II) is promoted more than in the GT with mean shear (Run III).

To show the difference in the mean chemical reaction rate between the three flows, the streamwise distributions of the mean concentrations of species $A$ and the chemical product $P, \overline{C_{A}}$, and $\overline{C_{P}}$, in the reacting case are shown in Fig. 6 . The mean concentrations are normalized by the initial concentration of species $A, C_{A 0}$. The mean concentration of the chemical product $P, \overline{C_{P}}$, is found from 

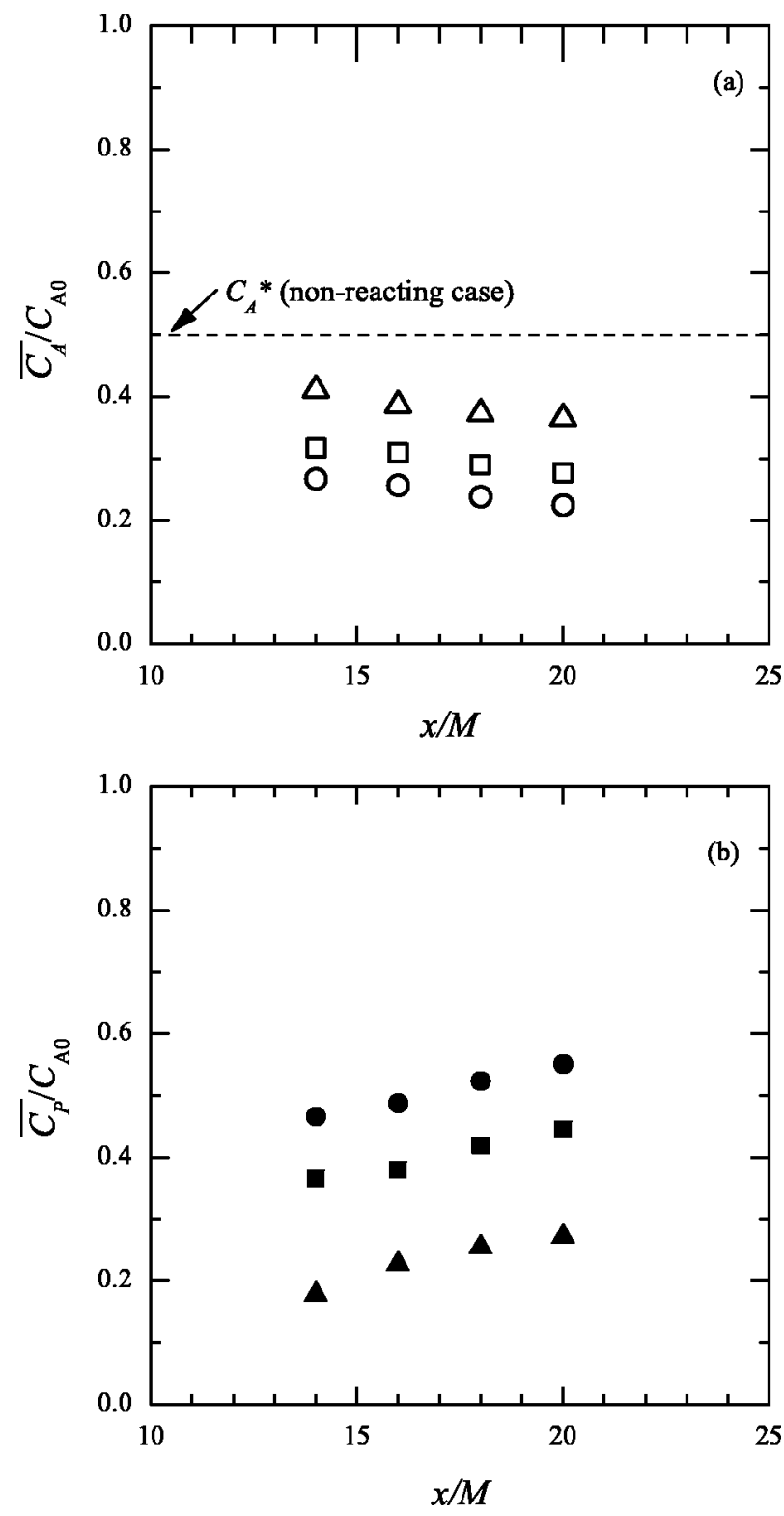

FIG. 6. Streamwise distributions of the mean concentrations of (a) species $A$ and (b) chemical product $P$ at $y / M=0.0: \triangle, \overline{C_{A}} / C_{A 0}$ in the pure GT; $\mathbf{\Delta}$, $\overline{C_{P}} / C_{A 0}$ in the pure GT; $\bigcirc, \overline{C_{A}} / C_{A 0}$ in the GT with ultrasound; $\bullet, \overline{C_{P}} / C_{A 0}$ in the GT with ultrasound; $\square, \overline{C_{A}} / C_{A 0}$ in the GT with mean shear; $\overline{C_{P}} / C_{A 0}$ in the GT with mean shear.

$$
\overline{C_{P}} / C_{A 0}=2\left(\overline{C_{A}^{*}}-\overline{C_{A}}\right) / C_{A 0},
$$

where $C_{A}^{*}$ is the concentration of the nonreacting species $A$. In order to derive Eq. (3), we used the following three experimental conditions mentioned in Sec. II.

(i) The reaction does not affect the flow field. That is, the reaction rate constant and fluid properties do not change depending on the reaction.

(ii) Molecular diffusivities of reacting and nonreacting species $A$ and $B$ have the same value.

(iii) The initial concentrations of species $A$ and $B$ have the same value.

The details of the derivation are described by Komori et al. ${ }^{16}$
It is confirmed that the progress of the chemical reaction results in the decrease of species $A$ and the increase of chemical product $P$ in the streamwise direction. The trends are more notable in the GT with ultrasound (Run II) than in the GT with mean shear (Run III). Figure 7 shows the vertical distributions of the mean concentration of chemical product $P$ obtained at $x / M=18$ together with those of species $A$. In the GT with ultrasound (Run II) and the GT with mean shear (Run III), the vertical gradients of the mean concentration of nonreacting species $A$ are gentle, compared to that in the pure GT (Run I). This means that turbulent mixing is enhanced by ultrasonic irradiation or mean fluid shear. The vertical distribution of the mean concentration of nonreacting species $A$ in the GT with ultrasound (Run II-NR) is close to that in the GT with mean shear (Run III-NR). Furthermore, it was found from the mass balance in the region of $0 \leqslant x / M$ $\leqslant 18$ that the two ultrasound and mean-sheared cases have almost the same amount of the nonreacting chemical species $A$ vertically transported by turbulent motions across the horizontal plane of $y / M=0$ in the region of $0<x / M<18$. This also means that the turbulent mass transport rate in the vertical direction is set to be almost equivalent between the two flows, as mentioned in Sec. II. Despite the almost the same amounts of the vertically transported reactant in Runs II and III, the chemical reaction is more promoted in the whole region of $y / M$ in the GT with ultrasound (Run II-R) than in the GT with mean shear (Run III-R). This is in agreement with the trends seen in the streamwise distributions of mean concentrations in Fig. 6.

To compare quantitatively the effects of the ultrasound and mean shear on the chemical reaction, the mean concentration of the chemical product $P$ at $x / M=18$ was integrated along the vertical $(y)$ axis $^{2}$ by

$$
P_{T}=\int_{-\infty}^{\infty} \frac{\overline{C_{P}}(y)}{C_{A 0}} d y .
$$

Here, it should be noted that $\overline{C_{P}}(y)$ and $\left|\bar{U}(y)-\overline{U_{a}}\right|$ have symmetric vertical distributions with respect to the horizontal plane of $y / M=0$ in the GT with means shear (Run III). The total amount of the chemical product, $P_{T}$, is compared for the three reacting flows in Fig. 8. The value of $P_{T}$ in the GT with ultrasound (Run II) is 2.2 times larger than $P_{T 0}$ in the pure GT (Run I), whereas $P_{T}$ in the GT with mean shear (Run III) is 1.6 times larger. It is concluded that highfrequency ultrasound is more effective as a tool for promoting chemical reaction than is mean fluid shear.

Despite the larger vertical mass flux in the GT with mean shear (Run III) than in the GT with ultrasound (Run II) (see Fig. 5), the total amount of the chemical product in the GT with ultrasound (Run II) is much larger than that in the GT with mean shear (Run III). To explore this, we calculated the power spectra of the vertical velocity fluctuation, $v$, and the cospectra of the vertical velocity fluctuation, $v$, and the concentration fluctuation of nonreacting species $A, c_{A}$. The power spectra and the cospectra multiplied by the frequency $f$ are shown at $y / M=0$ and $y / M=-0.6$ in Figs. 9 and 10, respectively. The area bounded by a cospectrum curve corresponds to the vertical mass flux in nonreacting condition. 

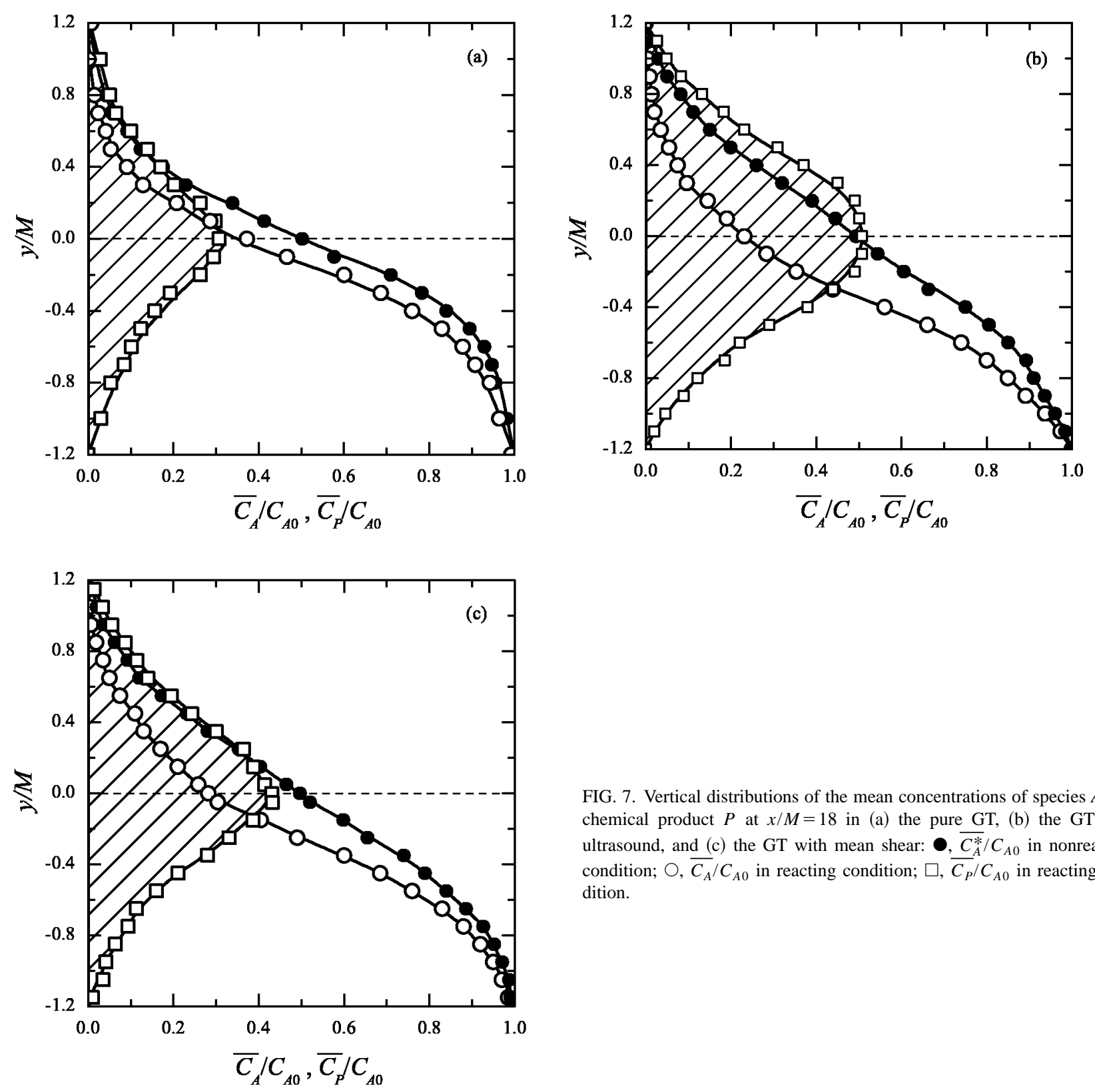

FIG. 7. Vertical distributions of the mean concentrations of species $A$ and chemical product $P$ at $x / M=18$ in (a) the pure GT, (b) the GT with ultrasound, and (c) the GT with mean shear: $\bullet, \overline{C_{A}^{*}} / C_{A 0}$ in nonreacting condition; $\bigcirc, \overline{C_{A}} / C_{A 0}$ in reacting condition; $\square, \overline{C_{P}} / C_{A 0}$ in reacting condition.

The vertical turbulent motions are enhanced at all scales in both the GT with ultrasound (Run II) and the GT with mean shear (Run III). Particularly in the GT with mean shear (Run III), the vertical fluctuation is more strongly promoted in the range of $0.5 \mathrm{~Hz} \leqslant f \leqslant 5-7 \mathrm{~Hz}$ than in the GT with ultrasound (Run II). But there the mass transfer is not always larger than that in the GT with ultrasound (Run II) and it becomes rather smaller in the high-frequency region of $f \geqslant 7 \mathrm{~Hz}$ even on the centerline with the larger vertical turbulence intensity. On the other hand, the mass transfer in the GT with ultrasound (Run II) is promoted at all scales and vertical turbulence intensity in the region away from the centerline overcomes the intensity at small scales in the sheared case (Run III) as shown in Fig. 9(b). In other words, the ultrasound deforms the interface between two reactive fluids up to the small scales, and complicatedly distributes the deformed interface over the wide region between the centerline and the walls, compared

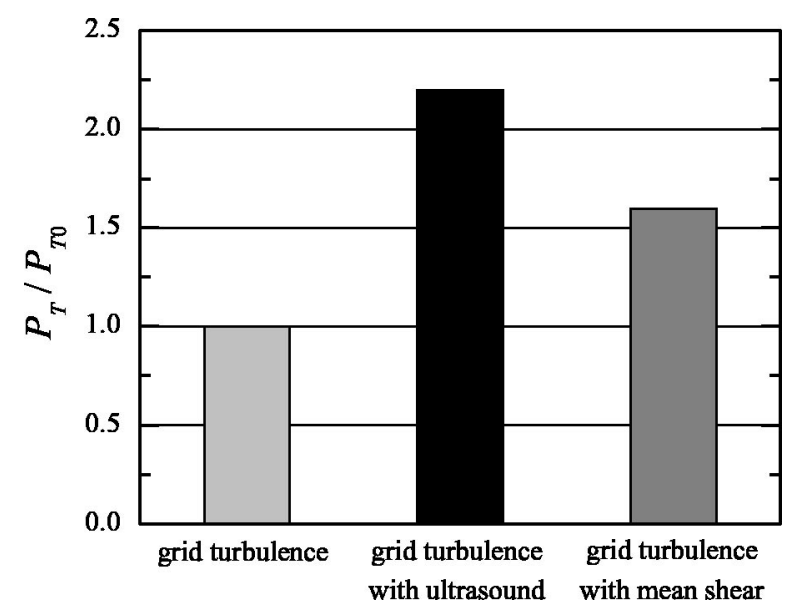

FIG. 8. Comparison of the amounts of chemical product $P$ at $x / M=18$ in the three reacting flows. 

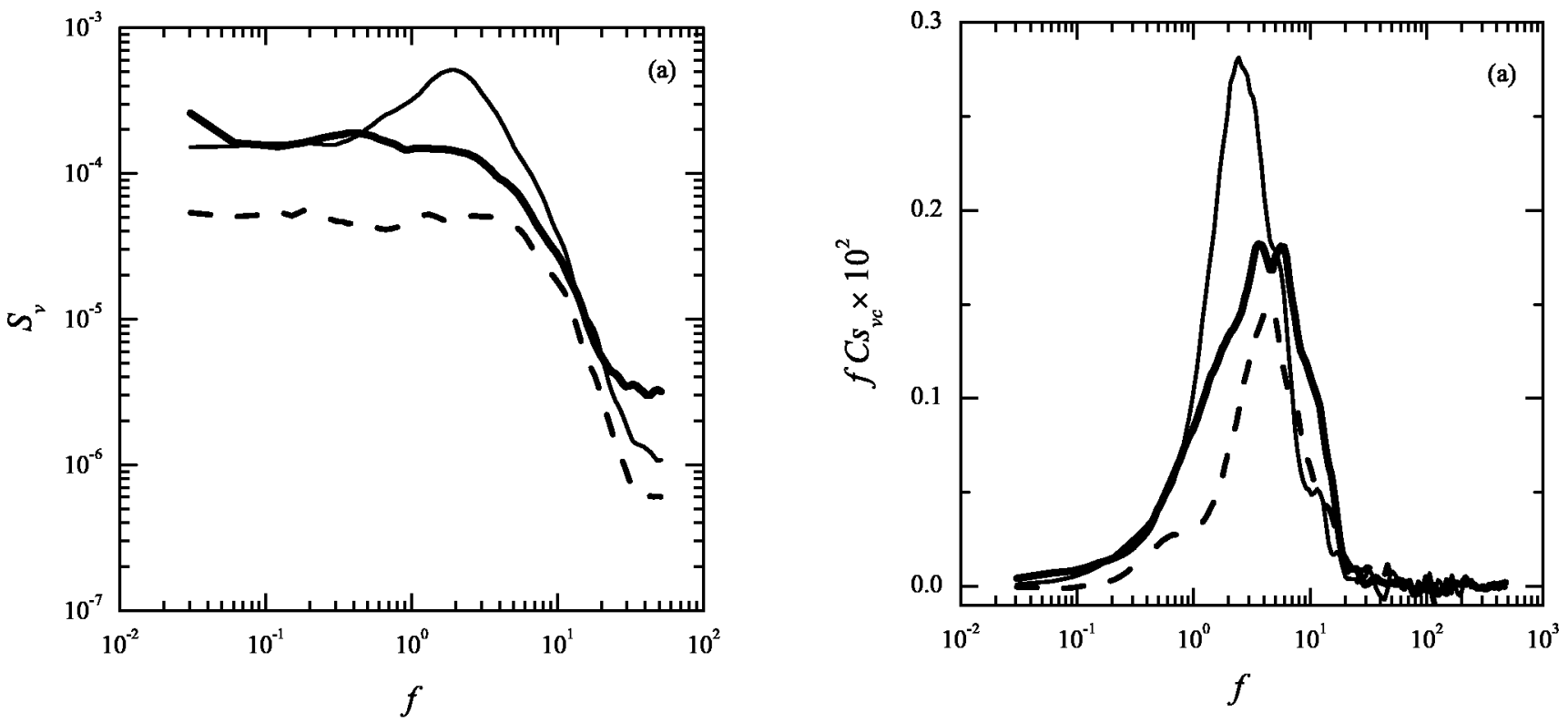

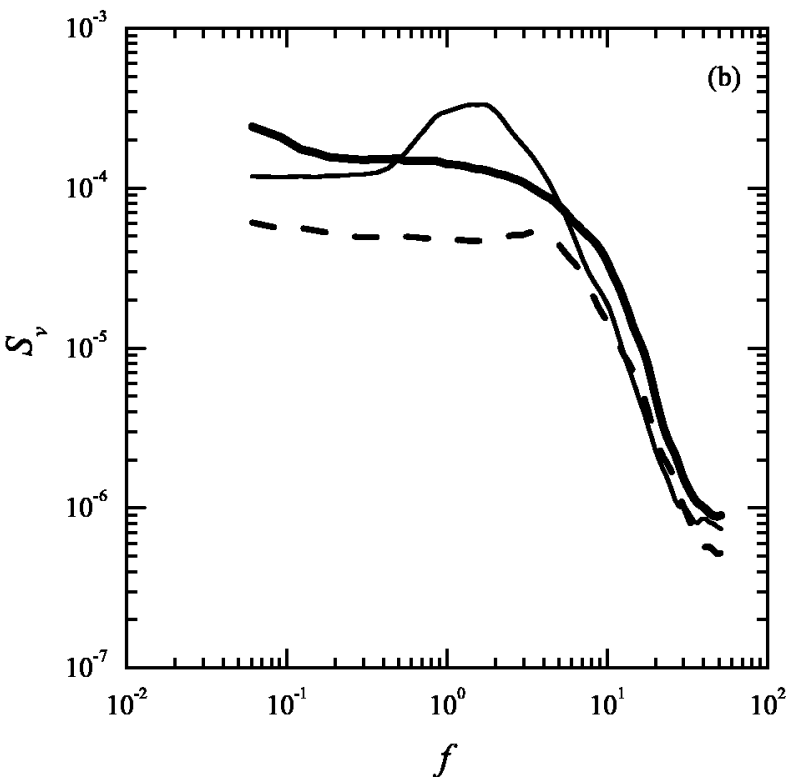

FIG. 9. Power spectra of vertical velocity fluctuation; (a) $x / M=18$ and $y / M=0.0$, (b) $x / M=18$ and $y / M=-0.6$. --- , in the pure GT (Run I); $\longrightarrow$, in the GT with ultrasound (Run II); - , in the GT with mean shear (Run III)

to the sheared case. Therefore, the chemical reaction progresses more rapidly in the GT with ultrasound (Run II) than that in the GT with mean shear (Run III).

Though the vertical turbulence intensity in the GT with mean shear (Run III) is larger or almost the same on the centerline of $y / M=0$ in the range $0.5 \mathrm{~Hz}<f<20 \mathrm{~Hz}$ than in the GT with ultrasound (Run II), as shown in Fig. 9(a), the vertical mass flux in the GT with mean shear (Run III) is smaller in the range $5 \mathrm{~Hz}<f<20 \mathrm{~Hz}$ than in the GT with ultrasound (Run II), as shown in Fig. 10(a). The reason why the vertical mass flux does not follow the vertical turbulence intensity in the GT with mean shear (Run III) especially on the central region can be explained as following. In the GT with mean shear (Run III), large-scale eddies are directly

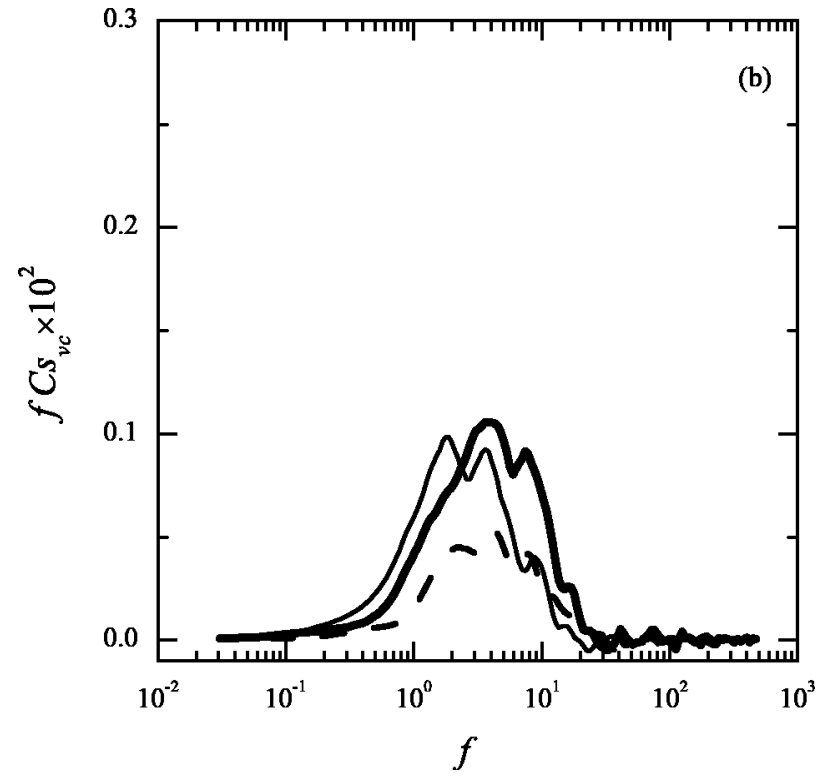

FIG. 10. Cospectra of vertical velocity fluctuation and concentration fluctuation of species $A$ in nonreacting condition; (a) $x / M=18$ and $y / M=0.0$, (b) $x / M=18$, and $y / M=-0.6$. Lines as in Fig. 9 .

generated by mean fluid shear and smaller-scale eddies are mainly generated in the central region through the cascade process. Therefore, the small-scale eddies generated by mean fluid shear are not always distributed in the interfacial region between the two reactants where the mass transfer rate is large, but the small-scale eddies mainly prevail in the central region around $y / M=0$ where the species $A$ and $B$ have been fully mixed by vortical motions in the upstream region. This results in no increase of the vertical mass flux on the centerline in the frequency range of $5 \mathrm{~Hz}<f<20 \mathrm{~Hz}$ and the equivalent mass flux at small scales to that the pure GT (Run I). On the other hand, the ultrasound generates the eddies with both large and small scales in the whole region between the centerline and the walls as shown in Fig. 4(a), and the strongly deformed interfacial area of species $A$ and $B$ widely 

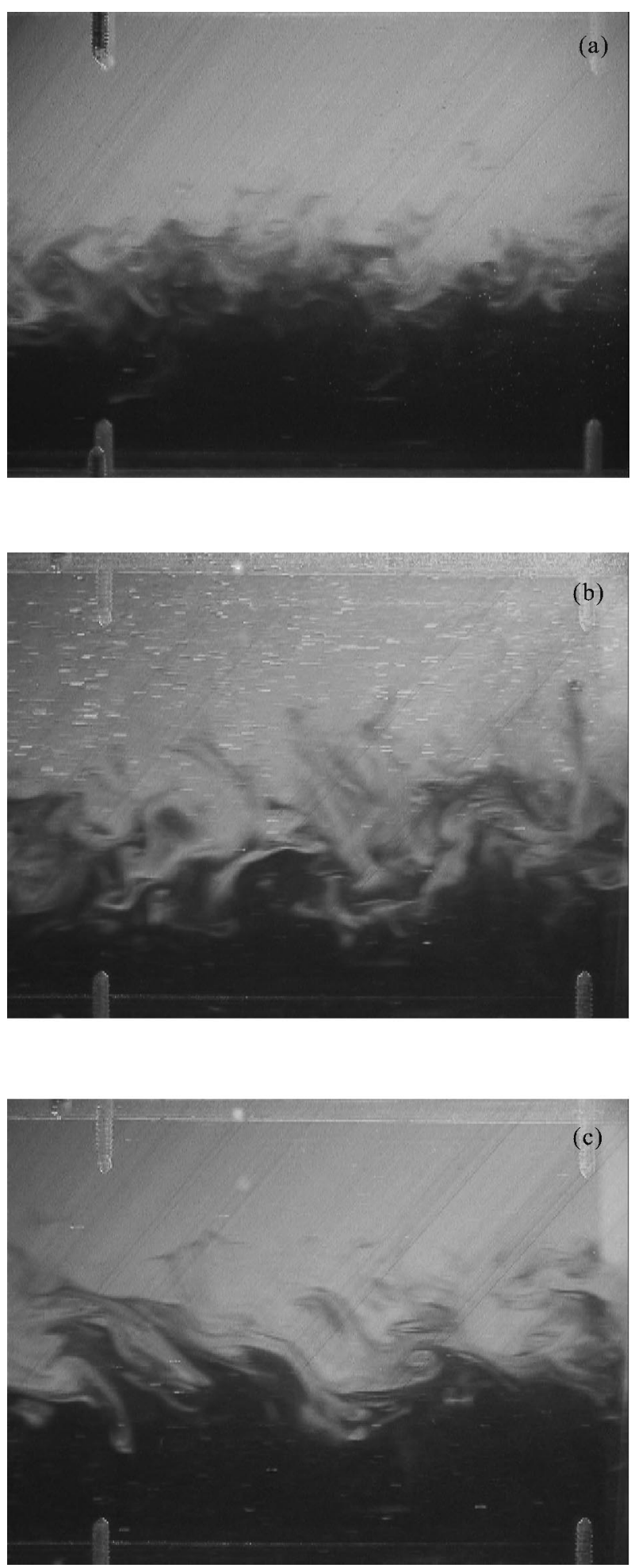

FIG. 11. Images of turbulent mixing in the nonreacting flow in the region of $x / M=15.5-19.0$; (a) mixing in the pure GT (Run I-NR), (b) mixing in the GT with ultrasound (Run II-NR), (c) mixing in the GT with mean shear (Run III-NR).

distributes not only in the central region but also in the outer region. This results in the increase of the vertical mass flux and strong promotion of chemical reaction owing to turbulent mixing at small scales in the GT with ultrasound (Run II).

These results on turbulent mixing can also be confirmed by visualizing the flows. Figure 11 shows images of turbulent mixing for the three flows in the nonreacting cases. The photographs were taken in the region of $15.5 \leqslant x / M \leqslant 19.0$ and the flow direction was from right to left. It is found that in the GT with ultrasound (Run II-NR) the interface between species $A$ and $B$ is expanded in the vertical direction and the species are well mixed at fine scales. On the other hand, in the GT with mean shear (Run III-NR), the vertical mass transfer is obviously promoted, but the scales of the sheargenerated eddies are larger than those in other flows and the species are less well mixed.

\section{CONCLUSIONS}

The effects of high-frequency ultrasound and mean fluid shear on turbulent mixing with a rapid chemical reaction were experimentally investigated in three types of turbulent liquid mixing-layer flow downstream of a turbulencegenerating grid. The main results obtained from this study can be summarized as follows.

Despite the enhancement of turbulent mass transport rate at an equivalent level, the difference in the turbulent mixing details between two reacting flows with ultrasonic irradiation and mean fluid shear considerably affects the progress of chemical reaction. In grid-generated turbulence with ultrasonic irradiation, the reactants are transferred at both large and small scales, so that the area of interface between two reactive fluids is enlarged. As a result, chemical reaction is strongly enhanced by ultrasonic irradiation. In grid-generated turbulence with mean fluid shear, the turbulent mixing is enhanced by mean fluid shear mainly at large scales, and therefore chemical reaction is not enhanced as much as in the grid-generated turbulence with ultrasonic irradiation. Thus the use of high-frequency ultrasound is a more promising technique for promoting turbulent mixing and chemical reaction than the use of mean fluid shear.

\section{ACKNOWLEDGMENTS}

This research was supported by the Proposal-Based New Industry Creative Type Technology R\&D Promotion Program (No. 99E 04-001-1) from the New Energy and Industrial Technology Development Organization (NEDO) of Japan. This research was also supported by a Grant-in-Aid for Scientific Research (B) (No. 11555059) from Japan Society for the Promotion of Science (JSPS).

${ }^{1}$ S. Komori, J. C. R. Hunt, T. Kanzaki, and Y. Murakami, "The effects of turbulent mixing on the correlation between two species and on concentration fluctuation in non-premixed reacting flows," J. Fluid Mech. 228, 629 (1991).

${ }^{2} \mathrm{~K}$. Nagata and S. Komori, "The effects of unstable stratification and mean shear on the chemical reaction in grid turbulence," J. Fluid Mech. 408, 39 (2000).

${ }^{3}$ H. Monnier, A. M. Wilhelm, and H. Delmas, "The influence of ultrasound on micromixing in a semi-batch reactor," Chem. Eng. Sci. 54, 2953 (1999).

${ }^{4}$ N. Kawahara, A. L. Yarin, G. Brenn, O. Kastner, and F. Durst, "Effect of acoustic streaming on the mass transfer from a sublimating sphere," Phys. Fluids 12, 912 (2000)

${ }^{5}$ N. P. Vichare, P. R. Gogate, V. Y. Dindore, and A. B. Pandit, "Mixing time analysis of a sonochemical reactor," Ultrason. Sonochem. 8, 23 (2001). 
${ }^{6}$ P. Vainshtein, M. Fichman, K. Shuster, and C. Gutfinger, "The effect of centerline particle concentration in a wave tube," J. Fluid Mech. 306, 31 (1996).

${ }^{7}$ P. Vainshtein, "Rayleigh streaming at large Reynolds number and its effect on shear flow," J. Fluid Mech. 285, 249 (1995).

${ }^{8}$ L. N. Liebermann, "The second viscosity of liquids," Phys. Rev. 75, 1415 (1949).

${ }^{9}$ H. Mitome, T. Kozuka, and T. Tuziuti, "Measurement of the establishment process of acoustic streaming using laser Doppler velocimetry" Ultrasonics 34, 527 (1996).

${ }^{10}$ T. Kamakura, T. Sudo, K. Matsuda, and Y. Kumamoto, "Time evolution of acoustic streaming from a planar ultrasound source," J. Acoust. Soc. Am. 100, 132 (1996).

${ }^{11}$ R. Clift, J. R. Grace, and M. E. Weber, Bubbles, Drops, and Particles (Academic, New York, 1978), pp. 97-139.

${ }^{12}$ H. Stapountzis, B. L. Sawford, J. C. R. Hunt, and R. E. Britter, "Structure of the temperature field downwind of a line source in grid turbulence," J. Fluid Mech. 165, 401 (1986).

${ }^{13}$ K. Nagata and S. Komori, "The difference in turbulent diffusion between active and passive scalars in stable thermal stratification," J. Fluid Mech. 430, 361 (2001)

${ }^{14}$ S. Komori, T. Kanzaki, and Y. Murakami, "Simultaneous measurements of instantaneous concentrations of two reacting species in a turbulent flow with a rapid reaction," Phys. Fluids A 3, 507 (1991).

${ }^{15}$ S. Komori and K. Nagata, "Effects of molecular diffusivities on countergradient scalar and momentum transfer in strongly stable stratification," J. Fluid Mech. 326, 205 (1996).

${ }^{16}$ S. Komori, K. Nagata, T. Kanzaki, and Y. Murakami, "Measurements of mass flux in a turbulent liquid flow with a chemical reaction," AIChE J. 39, 1611 (1993).

${ }^{17}$ R. S. Rogallo and P. Moin, "Numerical simulation of turbulent flows," Annu. Rev. Fluid Mech. 16, 99 (1984). 